\title{
Implant Migration and Functional Outcome of Reverse Shoulder Lateralized Glenosphere Line Extension System: A Study Protocol for a Randomized Controlled Trial
}

\section{Marie Louise Jensen ( $\nabla$ marie.louise.jensen.02@regionh.dk)}

Gentofte Hospital https://orcid.org/0000-0003-4489-3771

\section{Bo Sanderhoff Olsen}

Gentofte University Hospital: Gentofte Hospital

Marc Randall Kristensen Nyring

Gentofte University Hospital: Gentofte Hospital

Müjgan Yilmaz

Gentofte University Hospital: Gentofte Hospital

\section{Michael Mørk Petersen}

Rigshospitalet

\section{Gunnar Flivik}

Skåne University Hospital Lund: Skanes universitetssjukhus Lund

\section{Jeppe Vejlgaard Rasmussen}

Gentofte University Hospital: Gentofte Hospital

\section{Research Article}

Keywords: Rotator cuff arthropathy, reverse shoulder arthroplasty, lateralized glenosphere, scapular notching, radiostereometric analysis, clinical outcome

Posted Date: March 8th, 2022

DOI: https://doi.org/10.21203/rs.3.rs-1345634/v1

License: (c) (1) This work is licensed under a Creative Commons Attribution 4.0 International License. Read Full License 


\section{Abstract}

\section{Background}

Inferior scapular notching is a complication unique to reverse shoulder arthroplasty. The most efficient technique to avoid inferior scapular notching has been reported to be lateralization of the glenoid offset. This study aims to compare radiological and functional outcomes of the DELTA Xtend ${ }^{\circledR}$ Reverse Shoulder System Lateralized Glenosphere Line Extension (intervention group) with the Standard DELTA Xtend ${ }^{\circledR}$ Reverse Shoulder System (control group).

\section{Methods}

In this randomized controlled trial, all Danish citizens with rotator cuff arthropathy or degeneration of the glenohumeral joint with severe posterior wear and allocated for a reverse total shoulder arthroplasty at the department of orthopaedic surgery at Herlev and Gentofte Hospital, Copenhagen University Hospital will be considered for participation. The exclusion criteria are: Below 50 years of age; Cognitive or linguistic impairment; Insufficient glenoid bone stock; Previous fracture in the upper extremity; Autoimmune mediated inflammatory arthritis. There will be included a total of 122 patients of which 56 will participate in the radiostereometric analysis. This number of patients allows $20 \%$ to drop out. The primary outcome is the pattern and magnitude of the migration of the glenoid component and functional outcome (WOOS score). The secondary outcomes are the position of the arthroplasty, loosening, inferior scapular notching, patient-reported and functional outcomes (Oxford shoulder score, Constant Murley score and pain), number of complications, readmissions, revision, changes in bone mineral density and economy. The migration is assessed by radiostereometric analysis and the bone mineral density. The included patients will be examined before the surgery, within 1 week and 3, 6, 12 and 24 months after.

\section{Discussion}

No previous studies have compared the conventional reverse shoulder arthroplasty with the lateralized reverse shoulder arthroplasty in a randomized controlled trial regarding functional, radiographic and patient-reported outcomes. Furthermore, radiostereometric analysis has not been used to evaluate the migration of reverse shoulder arthroplasty. This study intends to determine which treatment is best for the benefit of future patients with an indication for reverse shoulder arthroplasty.

\section{Trial registration}

The study has been notified to Pactius and has approval number P-2021-231. Furthermore, the study will be registered on clinicaltrials.gov before start inclusion.

\section{Background}

Reverse total shoulder arthroplasty is the preferred implant for patients with degeneration of the glenohumeral joint and wear or tear of at least one rotator cuff tendon $[1,2]$ and the aim of the surgery is 
pain relief, improved range of motion and relatively low risk of revision surgery [3, 4].

Inferior scapular notching, which is damage to the inferior part of the bone of the glenoid component, is a complication unique to reverse shoulder arthroplasty and was described and graded by Sirveaux et al. in 2004 (4) and reported that $63 \%$ of the patients showed signs of inferior scapular notching. A more recent study (5) reported inferior scapular notching in $10 \%$ of 476 reverse shoulder arthroplasty implants and found lower clinical outcome scores, lower strength and range of motion (ROM) when present. Both studies found higher revision rates in this subgroup of patients $[4,5]$. Furthermore, Roche et al. (6) reported that inferior scapular notching may decrease glenoid baseplate stability which might ultimately lead to aseptic loosening in the clinical situation.

Different techniques to avoid inferior scapular notching have been reported (7). Lawrence et al (8) reported that the most efficient technique was to use a lateral offset of the glenoid component. Previous studies have found that a lateralized design of the reverse shoulder arthroplasty led to improved rotation and a lower risk of scapular notching $[9,11]$. The offset can be lateralized either biologically or with metal augmentation $(10)$. Both bone graft lateralization $(9,10)$ and metallic lateralization $(9)$ have shown good outcomes according to function and pain relief. Both advantages and disadvantages have been mentioned in relation to the two types of lateralization (7). To our knowledge the two types of lateralization have not been directly compared in a prospective randomized set-up.

Theoretically, the lateralized design can be associated with a greater risk of loosening of the glenoid component due to the tilt forces $(9,10)$. However, some studies have shown good outcomes according to glenoid loosening after lateralization (7) and others show varying results (9). Still, the consequences of using a lateralized design are poorly described.

Plain x-rays are unable to detect minor implant migration and authors have recommended that radiostereometric analysis should be used instead $[12,13]$. By inserting small tantalum beads into the surrounding bone, implant migration can be measured extremely accurate (14). Technical advances within radiostereometric analysis have made it possible to identify the implant and its position using the geometry of the implant instead of attaching tantalum beads to the implant (model-based radiostereometric analysis). The model-based radiostereometric analysis technique is less precise than the marker-based method. However, precision error values are still acceptable for clinical studies aimed at evaluating implant migration (15). The use of radiostereometric analysis for the evaluation of implant migration has been used frequently in the evaluation of hip and knee arthroplasty surgery. It has been shown that the late revision due to aseptic loosening of the tibial component in total knee arthroplasty is consistent with early radiostereometric analysis findings of continuous migration past the first postoperative year (16). Therefore, it has been suggested that a small series of new arthroplasties should be monitored with radiostereometric analysis the first 2 years postoperatively, as part of a safe phased introduction of new arthroplasties (17-19).

In shoulder arthroplasty surgery, radiostereometric analysis has been used to study the migration of the glenoid component in anatomical total shoulder arthroplasty $[20,21]$ and, in a few cases, migration of 
hydroxy-coated resurfacing humeral components [22, 23]. Previous studies have used radiostereometric analysis to access migration of the reverse shoulder arthroplasty $[24,25]$. To our knowledge, conventional reverse shoulder arthroplasty and lateralized reverse shoulder arthroplasty systems have not been compared in a randomized design regarding the clinical outcome and patient-reported outcome.

This randomized controlled trial aims to compare the DELTA Xtend $\circledast$ Reverse Shoulder Lateralized Glenosphere Line Extension System (intervention group) to the Standard Delta Xtend® Reverse Shoulder System (control group) (Depuy Synthes, Raynham, MA, USA). We hypothesize that the lateralization decreases the risk of inferior scapular notching without decreasing the functional outcome of the patients evaluated with passive range of motion (PROM).

\section{Methods And Design}

\section{Study design}

This study is an investigator initiated, single-centre, 1:1 randomized controlled trial, which will compare the DELTA Xtend ${ }^{\circledR}$ Reverse Shoulder Lateralized Glenosphere Line Extension (intervention group) with the standard DELTA Xtend ${ }^{\circledR}$ Reverse Shoulder System (control group). The two implants are produced by Depuy Synthes (Raynham, MA, USA). The SPIRIT reporting guidelines (26) and checklist (appendix 1) was used.

\section{Method}

Inclusion Criteria:

1. Rotator cuff arthropathy defined as degeneration of the glenohumeral joint and wear or tear of at least one rotator cuff tendon

2. Degeneration of the glenohumeral joint with intact rotator cuff function but severe posterior wear of the glenoid ( $>20$ degrees posterior wear)

3. Insufficient effect of non-surgical treatment with symptoms severe enough to justify shoulder arthroplasty.

4. ASA (American Society of Anesthesiology) score 1-3, physically fit for surgery and rehabilitation

Exclusion criteria:

1. Below 50 years of age

2. Cognitive or linguistic impairment

3. Insufficient glenoid bone-stock

4. Previous fracture in the upper extremities

5. Patients with autoimmune mediated inflammatory arthritis

6. Glenoid border medial to the medial border of the coracoid on a true AP radiograph (Figure 1) 


\section{Enrolment}

All Danish citizens with the diagnosis of rotator cuff arthropathy (degeneration of the glenohumeral joint and wear or tear of at least one rotator cuff tendon) or degeneration of the glenohumeral joint with severe posterior wear with an indication of a reverse total shoulder arthroplasty referred to the orthopaedic department at Herlev and Gentofte Hospital, Copenhagen University Hospital will be considered for participation in the trial. The medical records will be reviewed by the treating surgeon, who will evaluate if the patient can participate in the study, based on the above-mentioned inclusion and exclusion criteria. The patients will not be included in the study if the glenoid surface is medial to the medial border of the coracoid process on a true AP x-ray. The measurement is done by the following steps on true AP x-ray: 1) The coracoid process is identified 2) A reference line is drawn horizontal on the acromion 3) A perpendicular line is drawn to the acromion reference line, passing through the lateral side of the coracoid process base 4) A perpendicular line is drawn to the acromion reference line passing through the medial side of the coracoid process base 5) The glenoid base is identified, Figure 1. If the glenoid surface is medial to the medial border of the coracoid process on true AP a bone graft technique should be considered.

If the criteria are fulfilled, the treating surgeon will give the information (Appendix $2+3$ ) to the responsible investigator (Figure 2) and participation will be offered to the patient.

The patients who are interested in participation are invited for an undisturbed consultation with the treating surgeon. If the surgeon assesses the patient to be included in the study, the patient will receive written and oral information about the project. Additionally, the patient will be informed of the possibility to bring a bystander for the preparation consultation. The preparation consultation, held with the corresponding investigator, is minimum 24 hours later than the previous consultation, minimum 24 hours prior to the surgery and in an undisturbed room. The patient and a possible bystander will be re-informed of the study and the informed consent (Appendix 4) will be collected if the patient accepts to participate. Thereby the patient has had minimum 24 hours to reflect on the decision and the opportunity to bring a bystander.

The patients will then be asked to complete four questionnaires (Oxford Shoulder Score (OSS), Western Ontario Osteoarthritis of the Shoulder index (WOOS score), EQ- 5D-5L and pain with the visual analogue scale (VAS-score)). The informed consent gives the primary investigator access to information regarding medication, occupation, age, gender, comorbidity, and education, from medical records and questionnaires. Furthermore, the patients will have an examination with measurement of the activity of daily living, pain, strength (Constant-Murley score), range of movement and a radiographic examination with plain radiographs with standard anterior-posterior and lateral projections, MRI of the index shoulder joint and CT scan. The glenoid medialization will be measured by using true AP radiograph, Figure 1. Duel energy $\mathrm{x}$-ray absorptiometry (DXA) is used to assess bone mineral density (BMD) and osteopenia of the proximal humerus and distal forearm. 
The patients will get an extra radiation dosage of $0.10 \mathrm{mSV}$ including all the tests, which corresponds to the background radiation of 12 days in Denmark. In addition to the standard treatment the participating patients will have additional radiographic examinations including radiostereometric analysis, plain radiographs and DXA, Figure 3 and 4 .

\section{Randomization}

After the assessment of the sample calculation the study will aim towards including a total of 122 patients of which the first 56 patients will be examined with radiostereometric analysis and DXA. The patients are divided into two, equally sized and equally randomized to both arms, groups:

1. DELTA Xtend Reverse Shoulder System Lateralized Glenosphere Line Extension (intervention group)

2. Standard DELTA Xtend Reverse Shoulder System (control group).

In the operation room the randomization is done on the trial laptop, short after the perioperative evaluation of the quality of bone stock. Thereby, the randomization is done when the patient is anaesthetized. The randomization done on the trial laptop is done in the Research Electronic Data Capture (REdCap), in which the computer generates the randomization sequence by block randomization, stratified for gender and age and equally randomized to both shoulders (1:1 allocation). An independent statistician prepares the randomization table.

\section{Blinding}

It is not possible to blind the observer because the implant migration is assessed using radiostereometric analysis. However, patient-reported outcomes are solely assessed by the patients before the follow-up examination, without involving the surgeon or observer. The patients will be blinded to their allocated treatment the first two years after surgery. The statistician, who performs the analysis, is blinded to the randomization.

\section{Surgical procedure}

The procedures will be performed at Herlev and Gentofte Hospital, Copenhagen University Hospital. This unit performs around 300 primary and revision shoulder arthroplasties each year. From the evaluation of the number of surgeries from 2015 to 2016, it is expected to perform surgery on 75 patients with reverse shoulder arthroplasty for rotator cuff arthropathy every year. To minimize a potential learning curve and ensure a high surgical standard, the study is performed as a single-centre study and six experienced shoulder surgeons will be performing this type of surgery regularly using the same implant. To determine the potential learning curve applied by the lateralization devices, there will be done subgroup analyses at the end of the study, to compare the first and second half of the patients. Prior to the initiation of the study, each of the six surgeons shall have performed a minimum of 3 surgeries with the DELTA Xtend Reverse Shoulder System Lateralized Glenosphere Line Extension to minimize a potential learning curve problem. 
The patient is under general anaesthesia and in a beach chair position during the procedure. Surgical technique, including soft tissue balancing and exposure instrumentation, is standardized for all patients. All surgeries are done with the standard deltopectoral approach and subscapularis tenodesis. All patients are treated with either 1) DELTA Xtend Reverse Shoulder System Lateralized Glenosphere Line Extension or 2) the standard DELTA Xtend Reverse Shoulder System according to the guidelines from the manufacturer. Perioperative approximately 20 tantalum markers $(0.8 \mathrm{~mm}$, Tilly Medical Products, Lund, Sweden) are placed in the bone at the proximal humerus and the glenoid around the components, we aim for the widest non-linear distribution, which will allow us to measure segmental motion with Mb-RSA software (Model-based RSA 4.1, 2003-2014 RSAcore Department of orthopedics Leiden University Medical Centre) using Computer-Aided Design (CAD) models.

There will be used size 1 and 2 humeral components, with which all patients are treated with a cemented humeral monobloc component. Based on the medialization measured by plain radiographs, there will be used a $4 \mathrm{~mm}$ or $8 \mathrm{~mm}$ lateralized glenosphere if randomized for intervention group. If randomized for the control group a standard glenosphere is used. Prophylactic Cloxacillin $2 \mathrm{~g}$ and benzylpenicillin $1.2 \mathrm{~g}$ are given preoperatively and at 6 and 12 hours. If a patient cannot tolerate the standard prophylactic treatment, cefuroxime $1.5 \mathrm{~g}$ is given preoperative and at 6 and 12 hours.

\section{Rehabilitation}

All patients will receive a standard rehabilitation program led by a physiotherapist. A physiotherapist will one day postoperative instruct the patients in oedema prophylaxis. The patient will use a sling for 2 weeks, after which the patient has an appointment with the physiotherapist at the hospital for instructions in non-weight bearing training. Subsequently, the patient has an appointment with the physiotherapist once a week. There is a minimum of 3 months of training, longer if needed. Weightbearing training is allowed after 6 weeks.

\section{Outcome measures}

\section{Primary radiological outcome}

Migration of the glenoid components is assessed by model-based radiostereometric analysis, which is performed according to the guidelines of Valstar and Colleagues (13). In this part of the study, the first 56 patients are included. Maximum Total Point Motion (MTPM) after 2 years of follow-up is compared to the baseline value and is used as the primary radiostereometric analysis effect parameter. The accuracy of radiostereometric analysis will be assessed by at least 12 double examinations. The reference examination will be done within 1 week after the surgery and the follow-up examinations are planned at 3 months, 6 months, 1 year and 2 years. Radiostereometric analysis is conducted using a uniplanar radiostereometric analysis arrangement (UmRSA®- Calibration Cage No 43 (hip, spine and shoulder)). The x-ray analysis is performed using the model-based radiostereometric analysis commercial software (RSAcore, Department of Orthopedics, Leiden University Medical Center, Leiden, The Netherlands), available at Skaane University Hospital, Lund Sweden, where the analyses of radiostereometric analysis- 
$\mathrm{x}$-rays will be conducted. The exact set-up for the radiostereometric analysis (e.g. various distances and the degree between the $2 \mathrm{x}$-ray tubes) will be specified from a small phantom study and measurements of the 6 pilot patients (3 with each of the 2 types of implants). 42 combined CAD models for MB-RSA will be delivered by Depuy Synthes (Raynham, MA, USA).

\section{Primary functional outcome}

WOOS score: The WOOS score is a disease-specific patient-reported outcome (27). It contains 19 questions, divided into four groups: Sports and work, physical symptoms, emotions and lifestyle. The questions are answered using a visual analogue scale ranging from 0 to 100 . The overall score is from 0 to 1900 , where 1900 is the worst result. The scores are converted into a percentage of the maximum score to ease the interpretation. The difference in mean score between the preoperative score and the 2year follow-up score is the primary value of interest. In this study, there is used a version of WOOS, which has been translated to Danish according to the guidelines of Guillemin, Bombardier and Beaton (28). This version has been validated with classical test theory in a cohort of patients treated with shoulder arthroplasty for osteoarthritis (29).

\section{Secondary radiological outcomes}

Plain radiographs: the plain radiographs will be taken preoperatively, in the first week postoperatively, at 3 months, 1 year and 2 years. There will be used an anterior-posterior and lateral view. The radiographs will be used for evaluation of the position of the component, inferior scapular notching and loosening as a cause of failure.

DXA: the first 56 patients will be included in this part of the study. DXA will be conducted preoperatively to evaluate BMD of the proximal humerus, scapula and distal forearm. BMD measurements of the distal forearm will be performed bilateral and used as reference to the shoulder and to adjust for changes in BMD that are not related to the shoulder arthroplasty. The difference in mean score between the preoperative BMD score and the 2-year follow-up score is the primary value of interest as it represents the change after the implant. The precision of DXA will be assessed by 12 double examinations.

\section{Secondary functional outcomes}

Constant-Murley Score: The Constant-Murley Score includes an evaluation of pain, range of motion, activities of daily living and strength. The maximum score for the evaluation of pain and activities of daily living is 35 points. For the objective evaluation there is a maximum score of 65 points, of which 40 are from a range of motion and 25 are from strength. Thereby there is a collected maximum of 100 points, which indicates a shoulder without any disabilities. The difference in mean score between the preoperative score and the 2-year follow-up score is the primary value of interest. In this study, a Danish version (30) of the modified Constant-score (31) is used.

Oxford Shoulder Score (OSS): the OSS was invented as a measurement tool to assess function and pain after elective shoulder surgery (32). It contains 12 questions with a score from 0 -4. The total score ranges 
from 0 to 48, with 48 as the best score. The scores are converted into a percentage of the maximum score to ease the interpretation. The difference in mean score between the preoperative score and the 2-year follow-up score is the primary value of interest. In this study, the Danish version, which has been translated and validated with the classical test theory (33), of OSS is used.

Complications and side effects: Any incident of medical (cardiovascular, embolism, pneumonia) and surgical complications (nerve damage, fracture, infection, instability, disposition of component and dislocation) will be noted, as well as revision surgery (removal or exchange of any component).

Pain and patient satisfaction: Pain is evaluated on the day of examination with the VAS score with scores from 0 to 10, with 10 points as the worst possible pain. The patients will describe the outcome on a 7point scale, with "much better" as the best and "much worse" as the worst. The difference in mean score between the preoperative score and the 2-year follow-up score is the primary value of interest.

Economic assessment: Quality-adjusted life years (QALY) is determined from the threshold of the acceptable cost-utility ratios for the health care system to use. In respectively Europe and England the thresholds are set at 30,000 Euros and $20,000-30,000$ pounds. The study will compare the cost-utility of the DELTA Xtend Reverse Shoulder System Lateralized Glenosphere Line Extension with the set thresholds and the cost-utility of the Standard DELTA Xtend Reverse Shoulder System. To estimate the QALY for each patient, there will be used EQ-5D-5L. The difference in mean score between the preoperative score and the 2-year follow-up score is the primary value of interest. There will be defined a cost model from medical records, registries, data from patients and unit costs from the Danish health care system. Information regarding usage and readmission of pain medication, length of hospital stay, and discharge destination will be noted.

\section{Follow-up}

The follow-up time for included patients is 2 years (figure 3 and 4) with assessment within 1 week and at 3 months, 6 months, 1 and 2 years from the day of the surgery and thereby randomization. There will be conceived data on complications including revision surgery from medical records and hospital database after 10 years.

\section{Protocol violations, revision and drop-out}

If the surgeon during the surgery regards it as impossible to insert a glenoid component, the surgery can be converted to a stemmed hemiarthroplasty using the DELTA Xtend humeral stem and a CTA head.

If patients drop out of the trial, it will be recorded along with the reason for drop-out. The patient in question will be included in the final mixed-effect model analysis, except for patients who drop out before 3 months, who cannot be included in the analysis. At the end of the study, a sensitivity analysis will be performed to evaluate the impact of missing data on the overall results of the trial. It will be recorded if there are indications that some patients do not comply with rehabilitation. 
In case of revision surgery, reason and new arthroplasty type will be recorded. Additionally, the patient will prior to revision surgery be assessed by DXA, radiostereometric analysis, patient-reported outcome and clinical outcome if possible. The patient will stay included in the study and the 2-year analysis with the latest follow-up.

Major protocol modifications will immediately be reported to all investigators and trial registries.

\section{Statistics}

Prior to the analysis, the nature of the data is assessed. It is expected to analyse the changes between pre- and postoperative assessment with paired parametric statistics. The differences between the groups are expected to be analysed using parametric statistics (student t-test). The radiostereometric analysis data is not expected to be normally distributed and will be analysed using non-parametric analysis of variance over time. The differences between these groups will be analysed with Mann-Whitney U-test. Because of the nature of repeated measures in this study, it is expected to use a mixed effects model to analyse the overall results.

\section{Sample size calculation radiostereometric analysis}

Sample size calculation for radiostereometric analysis (maximum total point motion of the glenoid component after 2 years will be used as primary radiostereometric analysis effect parameter) was performed with an expected standard deviation (SD) of $0.4 \mathrm{~mm}$, a minimally clinically important difference of $0.4 \mathrm{~mm}$, a significance level at $5 \%$ and power of 0.90 and resulted in 22 participants in each group (56 participants with allowance for 20 percent drop-out) (34). We expect to include 28 patients in the two randomized groups. However, we are aware of the possibility of dropouts and will be satisfied with a minimum of 22 patients in each group according to the power calculation. The estimation of SD = $0.4 \mathrm{~mm}$ is extrapolated from a study of migration of the Global CAP resurfacing arthroplasty(22).

\section{Sample size calculation WOOS}

The standard deviation of WOOS is set at 15 (15 percent of a maximum score). The minimally clinically important difference is set at 10 (10 percent of a maximum score), the significance level is set at $5 \%$ and the power of 0.90 . With these assumptions 48 patients are needed in each group resulting in a study population with 122 participants (34) (allowing for 20 percent drop-out).

Data on radiostereometric analysis and DXA will be analysed at the end of follow-up for the first 56 patients. No other interim data-analysis will be carried out.

\section{Discussion}

This study aims to determine if DELTA Xtend Reverse Shoulder System Lateralized Glenosphere Line Extension has a better outcome than the Standard DELTA Xtend Reverse Shoulder System. To do that there was made a sample size calculation and added 20 percent, to prevent the impact of dropouts. The 
patients will be divided into two groups and equally randomized and treated. There is however a possibility of being treated with an inferior arthroplasty, as it is not known if one is better as the two types of glenospheres are considered equal.

The standard follow-up time after a shoulder arthroplasty at our department is 3 months. The patients included in this study will additionally be examined within 1 week and at 6,12 and 24 months. This can both contribute to the patients feeling more secure by handling problems as they come or entail a longlasting feeling of being ill and furthermore being time-consuming. Sirveaux et al. (4) reported that inferior scapular notching was associated with increased risk of revision, and Mollon et al.(5) described lower clinical outcomes for these patients. This study aims to explore solutions to prevent inferior scapula notching and subsequently better outcomes. Lateralization of the glenoid off-set has earlier been reported as the most efficient technique to avoid inferior scapular notching (8). Furthermore, lateralization has been described to improve movement (9) but at the same time makes greater demands to the deltoid muscle (35). Nonetheless, there has also been reported a higher rate of loosening (8). Implant migration can be the first sign of loosening. Radiostereometric analysis has been used to measure implant migration in numerous studies for more than 20 years (18), but this is, to our knowledge, the first time radiostereometric analysis is used to compare the outcome for conventional reverse shoulder arthroplasty and lateralized reverse shoulder arthroplasty in a randomized controlled trial. DXA has been used in shoulder patients before. A previous study showed that two-thirds of elective shoulder arthroplasty patients had BMD meeting the criteria of treatment for osteoporosis (36). This study will further investigate the BMD for shoulder patients and if a shoulder arthroplasty affects it.

The study aims to earn knowledge of the best treatment option for future patients needing a reverse shoulder arthroplasty.

\section{Trial Status}

Version 1.0, January 24, 2022.

Start inclusion: April 1, 2022.

Finish date of recruitment to the first part of the study: March 31, 2023.

Finish date of recruitment of all 122 patients: March 31, 2024.

Finish date of follow-up for the first part of the study: March 31, 2025

Finish date of follow-up for all 122 patients: March 31, 2026

\section{Abbreviations}

ROM

Page $11 / 20$ 
Range of motion

PROM

Passive range of motion

ASA

American society of anaesthesiology

OSS

Oxford Shoulder Score

WOOS score

Western Ontario Osteoarthritis of the Shoulder index

VAS score

Visual analogue scale

DXA

Duel energy $\mathrm{x}$-ray absorptiometry

BMD

Bone mineral density

REdCap

Research Electronic data Capture

MB-RSA

Model-based radiostereometric analysis

CAD models

Computer-aided design models

MTPM

Maximum Total Point Motion

QALY

Quality adjusted life years

SD

Standard deviation.

\section{Declarations}

\section{Ethics approval and consent to participate}

The trial will be performed according to the Helsinki declaration (37). Before signing the informed consent form, all patients will receive oral and written information and have at least 24 hours and the possibility of a bystander. They can withdraw the consent at any time.

This protocol is approved by The Danish National Committee on Health Research Ethics with approval number $\mathrm{H}-21042181$.

\section{Consent for publication}

Not applicable. 


\section{Availability of data and materials}

The electronic data will be stored in closed drives and printed data will be stored in closed cabinets. The datasets used and/or analysed during the current study are available from the corresponding authors on reasonable request. The study has been notified to Pactius and has approval number P-2021-231.

\section{Competing interest}

The study is funded by the company Depuy Synthes (Raynham, MA, USA) and MP, JR and BO get institutional support from both Zimmer Biomet (Warsaw, IN, USA) and Depuy Synthes (Raynham, MA, USA). JR and BO have been paid to give lectures for Depuy Synthes (Raynham, MA, USA).

\section{Funding}

The study is partially funded by the manufacturer Depuy Synthes (Raynham, MA, USA) and will be supported with an amount of DKK 1,722,099. The research unit at Herlev and Gentofte Hospital has granted a scholarship covering 6 months' salary. The funders have no role in the design of the study, execution, analysis or interpretation, and the funders do not influence the decision to submit the results.

\section{Author contributions}

MJ: study design, performs the model-based radiostereometric analysis radiographs and DXA-scan and analyses model-based radiostereometric analysis x-rays and DXA-scans, data collection, data analysis, writing of the draft paper. BO: Study design, revision of papers, inclusion and surgery of patients. MN: responsible for the supervision of model-based radiostereometric analysis and DXA including analysis of DXA-scans. MY: responsible for the supervision of model-based radiostereometric analysis and DXA including analysis of DXA-scans. MP: study design, responsible for the supervision of model-based radiostereometric analysis and DXA, revision of papers. GF: responsible for supervision regarding analysis of the model-based radiostereometric analysis radiographs with the calculation of implant migration. JR: study design, revision of papers, inclusion and surgery of patients. The ICMJE recommendation (38) will be followed and all authors will read and approve the final manuscript.

\section{Acknowledgements}

Not applicable

\section{Authors information}

${ }^{1}$ Department of Orthopedic Surgery, Herlev and Gentofte Hospital, Copenhagen University Hospital, Copenhagen, Denmark.

${ }^{2}$ Department of Clinical Medicine, University of Copenhagen, Copenhagen, Denmark 
${ }^{3}$ Department of Orthopedic Surgery, Rigshospitalet, Copenhagen University Hospital, Copenhagen, Denmark

${ }^{4}$ Department of Orthopedic Surgery, Skaane University Hospital, Lund University, Sweden

\section{Publication}

It is expected that articles from the study will be published in international orthopaedic journals and it will be presented at international and national scientific meetings.

\section{References}

1. Cvetanovich GL, Waterman BR, Verma NN, Romeo AA. Management of the Irreparable Rotator Cuff Tear. J Am Acad Orthop Surg. 2019;27(24):909-17.

2. Berliner JL, Regalado-Magdos A, Ma CB, Feeley BT. Biomechanics of reverse total shoulder arthroplasty. J Shoulder Elb Surg. 2015;24:150-60.

3. Bacle G, Nové-Josserand L, Garaud P, Walch G. Long-Term Outcomes of Reverse Total Shoulder Arthroplasty. J Bone Jt Surg. 2017;99:454-61.

4. Sirveaux F, Favard L, Oudet D, Huquet D, Walch G, Molé D. Grammont inverted total shoulder arthroplasty in the treatment of glenohumeral osteoarthritis with massive rupture of the cuff. Results of a multicentre study of 80 shoulders. J Bone Jt Surg. 2004;86-B:388-95.

5. Mollon B, Mahure SA, Roche CP, Zuckerman JD. Impact of scapular notching on clinical outcomes after reverse total shoulder arthroplasty: an analysis of 476 shoulders. J Shoulder Elb Surg. 2017;26(7):1253-61.

6. Roche CP, Stroud NJ, Martin BL, Steiler CA, Flurin PH, Wright TW, et al. The impact of scapular notching on reverse shoulder glenoid fixation. J Shoulder Elb Surg. 2013;22:963-70.

7. Friedman RJ, Barcel DA, Eichinger JK. Scapular Notching in Reverse Total Shoulder Arthroplasty. J Am Acad Orthop Surg. 2019;27(6):200-9.

8. Lawrence C, Williams GR, Namdari S. Influence of glenosphere design on outcomes and complications of reverse arthroplasty: A systematic review. Clin Orthop Surg. 2016;8:288-97.

9. Valenti P, Sauzières P, Katz D, Kalouche I, Kilinc AS. Do less medialized reverse shoulder prostheses increase motion and reduce notching? Clin Orthop Relat Res. 2011;469:2550-7.

10. Boileau P, Moineau G, Roussanne Y, O'Shea K. FRCSI. Bony Increased Offset-Reversed Shoulder Arthroplasty (BIO-RSA). JBJS Essent Surg Tech. 2011;469(9):2558-67.

11. Boileau P, Moineau G, Roussanne Y, O'Shea K. Bony increased-offset reversed shoulder arthroplasty Minimizing scapular impingement while maximizing glenoid fixation. Clin Orthop Relat Res. 2011;469:2558-67.

12. Kärrholm J, Gill RH, Valstar ER. The History and Future of Radiostereometric Analysis. Clin Orthop Relat Res. 2006;(448):10-21. 
13. Valstar ER, Gill R, Ryd L, Flivik G, Börlin N, Kärrholm J. Guidelines for standardization of radiostereometry (RSA) of implants. Acta Orthop. 2005;76(4):563-72.

14. Ten Brinke B, Beumer A, Koenraadt KLM, Eygendaal D, Kraan GA, Mathijssen NMC. The accuracy and precision of radiostereometric analysis in upper limb arthroplasty. Acta Orthop. 2017;88(3):320-5.

15. Trozzi C, Kaptein BL, Garling EH, Shelyakova T, Russo A, Bragonzoni L, et al. Precision assessment of model-based RSA for a total knee prosthesis in a biplanar set-up. Knee. 2008;15:396-402.

16. Ryd L, Albrektsson BEJ, Carlsson L, Dansgard F, Herberts P, Lindstrand A, et al. Roentgen stereophotogrammetric analysis as a predictor of mechanical loosening of knee prostheses. $\mathrm{J}$ Bone Jt Surg [Br]. 1995;77-B(3):377-83.

17. Nelissen RG, Pijls BG, Kärrholm J, Malchau H, Nieuwenhuijse MJ, Valstar ER. RSA and Registries: The Quest for Phased Introduction of New Implants. J bone Jt Surg. 2011;93(Suppl 3):62-5.

18. Kärrholm J. Radiostereometric analysis of early implant migration - A valuable tool to ensure proper introduction of new implants. Acta Orthop. 2012;83(6):551-2.

19. Valstar E, Kaptein B, Nelissen R. Radiostereometry and new prostheses. Acta Orthop. 2012;83(2):103-4.

20. Nuttall D, Haines JF, Trail II. A study of the micromovement of pegged and keeled glenoid components compared using radiostereometric analysis. J shoulder Elb Surg. 2007;16(3 Suppl):6570.

21. Nuttall $D$, Haines JF, Trail IA. The early migration of a partially cemented fluted pegged glenoid component using radiostereometric analysis. J Shoulder Elb Surg. 2012;21:1191-6.

22. Nuttall D, Birch A, Haines JF, Trail IA. Radiostereographic analysis of a shoulder surface replacement: does hydroxyapatite have a place? Bone Joint J. 2014 Aug;96-B(8):1077-81.

23. Mechlenburg I, Klebe TM, Døssing KV, Amstrup A, Søballe K, Stilling M. Evaluation of periprosthetic bone mineral density and postoperative migration of humeral head resurfacing implants: two-year results of a randomized controlled clinical trial. J shoulder Elb Surg. 2014 Oct;23(10):1427-36.

24. Fraser AN, Tsukanaka M, Fjalestad T, Madsen JE, Röhrl SM. Model-based RSA is suitable for clinical trials on the glenoid component of reverse total shoulder arthroplasty. J Orthop Res Off Publ Orthop Res Soc. 2018 Dec;36(12):3299-307.

25. Fraser AN, Bøe B, Fjalestad T, Madsen JE, Röhrl SM. Stable glenoid component of reverse total shoulder arthroplasty at 2 years as measured with model-based radiostereometric analysis (RSA). Acta Orthop. 2021 Jul; 1-7.

26. Chan A-W, Tetzlaff J, Gøtzsche P, Altman D, Mann H, Berlin J, et al. SPIRIT 2013 Explanation and Elaboration: Guidance for protocols of clinical trials. BMJ. 2013;346:e7586.

27. Lo IKY, Griffin S, Kirkley A. The development of a disease-specific quality of life measurement tool for osteoarthritis of the shoulder: The Western Ontario Osteoarthritis of the Shoulder (WOOS) index. J Osteoarthr Res Soc Int. 2001;9:771-8. 
28. Guillemin F, Bombardier C, Beaton D. Cross-cultural adaptation of health-related quality of life measures: literature review and proposed guidelines. J Clin Epidemiol. 1993;46(12):1417-32.

29. Rasmussen JV, Jakobsen J, Olsen BS, Brorson S. Translation and validation of the Western Ontario Osteoarthritis of the Shoulder (WOOS) index - the Danish version. Patient Relat Outcome Meas. 2013;4:49-54.

30. Ban I, Troelsen A, Christiansen DH, Svendsen SW, Kristensen MT. Standardised test protocol (Constant Score) for evaluation of functionality in patients with shoulder disorders. Dan Med J. 2013 Apr;60(4):A4608.

31. Constant CR, Gerber C, Emery RJH, Søjbjerg JO, Gohlke F, Boileau P. A review of the Constant score: modifications and guidelines for its use. J shoulder Elb Surg. 2008;17(2):355-61.

32. Dawson J, Fitzpatrick R, Carr A. Questionnaire on the perceptions of patients about shoulder surgery. J Bone Jt Surg. 1996;78-B(4):593-600.

33. Frich LH, Noergaard PM, Brorson S. Validation of the Danish version of Oxford Shoulder Score. Dan Med Bull. 2011 Nov;58(11):A4335.

34. Dhand N, Khatkar M Statulator: an online statistical calculator. Sample size calculator for comparing two independent means [Internet]. Statulator. 2014. Available from:

http://statulator.com/SampleSize/ss2M.\%0Ahtml\#.

35. Nolte P-C, Miles JW, Tanghe KK, Brady AW, Midtgaard KS, Cooper JD, et al. The effect of glenosphere lateralization and inferiorization on deltoid force in reverse total shoulder arthroplasty. J Shoulder Elb Surg. 2021;30(8):1817-26.

36. Bernatz JT, Brooks AE, Nguyen BP, Shin ED, Binkley NC, Anderson PA, et al. Prevalence and Treatment of Osteoporosis Prior to Elective Shoulder Arthroplasty. J Am Acad Orthop Surg Glob Res Rev. 2020 Dec;4(12):e20.00204.

37. WMA Declaration of Helsinki - Ethical Principles for Medical Research involving human subjects. 2018. https://www.wma.net/policies-post/wma-declaration-of-helsinki-ethical-principles-for-medicalresearch-involving-human-subjects/. Accessed 20 Jul 2021.

38. ICMJE Recommendations for the Conduct, Reporting, Editing, and Publication of Scholarly Work in Medical Journals. 2018. http://www.icmje.org/icmje-recommendations.pdf. Accessed 20 Jul 2021.

\section{Figures}




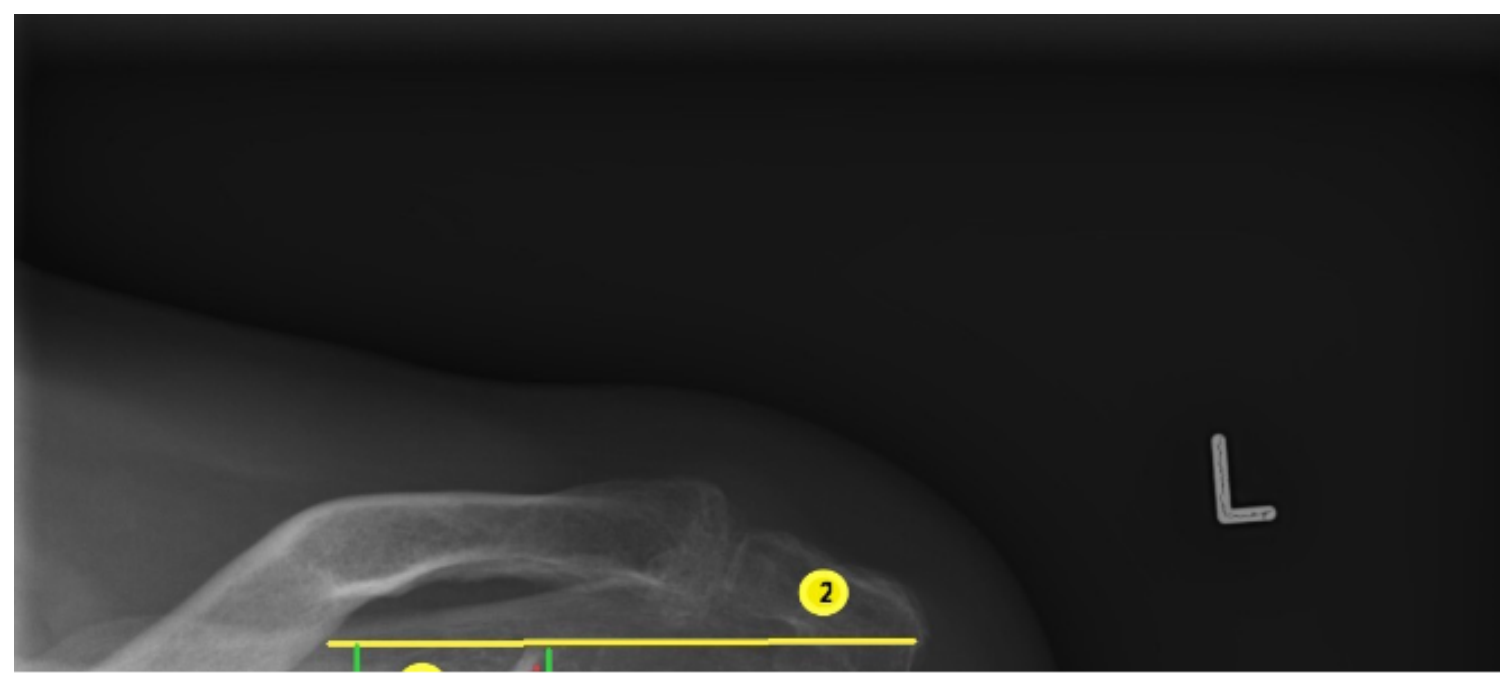

Figure 1

Assessment of medialization on true AP radiograph

Figure 2 
CONSORT flow diagram of the progress of the study

\begin{tabular}{|c|c|c|c|c|c|c|c|}
\hline & \multicolumn{7}{|c|}{ STUDY PERIOD } \\
\hline & Enrolment & Allocation & & & st-allocatio & & \\
\hline TIMEPOINT & $-t_{1}$ & 0 & 1 week & 3 months & 6 months & 1 year & 2 years \\
\hline ENROLMENT & & & & & & & \\
\hline $\begin{array}{l}\text { Eligibility } \\
\text { screen }\end{array}$ & $\mathrm{x}$ & & & & & & \\
\hline $\begin{array}{l}\text { Informed } \\
\text { consent }\end{array}$ & $x$ & & & & & & \\
\hline Allocation & & $x$ & & & & & \\
\hline INTERVENTION & & & & & & & \\
\hline $\begin{array}{l}\text { Lateralized } \\
\text { system }\end{array}$ & & $x$ & & & & & \\
\hline $\begin{array}{l}\text { Standard } \\
\text { system }\end{array}$ & & $\mathrm{X}$ & & & & & \\
\hline ASSESSMENT & & & & & & & \\
\hline$x$-ray & $\mathrm{x}$ & & & & & & \\
\hline CT & $\mathrm{x}$ & & & & & & \\
\hline$M R I$ & $\mathrm{x}$ & & & & & & \\
\hline$D X A$ & $x$ & & $x$ & $\mathrm{x}$ & $\mathrm{x}$ & $x$ & $\mathrm{X}$ \\
\hline$R S A$ & & & $\mathrm{x}$ & $\mathrm{x}$ & $x$ & $\mathrm{x}$ & $x$ \\
\hline $\begin{array}{l}\text { Constant- } \\
\text { Murley score }\end{array}$ & $x$ & & & $x$ & $x$ & $x$ & $x$ \\
\hline woos & $\mathrm{x}$ & & & $\mathrm{x}$ & $\mathrm{x}$ & $\mathrm{x}$ & $\mathrm{x}$ \\
\hline oss & $\bar{x}$ & & & $\bar{x}$ & $\mathrm{x}$ & $\bar{x}$ & $\bar{x}$ \\
\hline$E Q-5 D-5 L$ & $x$ & & & $\mathrm{x}$ & $x$ & $x$ & $x$ \\
\hline
\end{tabular}

Figure 3

SPIRIT figure. Schedule of enrolment and assessments of the 56 radiostereometric analysis patients. 


\begin{tabular}{|c|c|c|c|c|c|c|c|}
\hline & \multicolumn{7}{|c|}{ STUDY PERIOD } \\
\hline & Enrolment & Allocation & & & st-allocatio & & \\
\hline TIMEPOINT & $-t_{1}$ & 0 & 1 week & 3 months & 6 months & 1 year & 2 years \\
\hline ENROLMENT & & & & & & & \\
\hline $\begin{array}{c}\text { Eligibility } \\
\text { screen }\end{array}$ & $x$ & & & & & & \\
\hline $\begin{array}{l}\text { Informed } \\
\text { consent }\end{array}$ & $\mathrm{x}$ & & & & & & \\
\hline Allocation & & $\mathrm{X}$ & & & & & \\
\hline INTERVENTION & & & & & & & \\
\hline $\begin{array}{l}\text { Lateralized } \\
\text { system }\end{array}$ & & $\mathrm{x}$ & & & & & \\
\hline $\begin{array}{l}\text { Standard } \\
\text { system }\end{array}$ & & $\mathrm{X}$ & & & & & \\
\hline ASSESSMENT & & & & & & & \\
\hline$x$-ray & $x$ & & $x$ & & & $\mathrm{X}$ & $\mathrm{X}$ \\
\hline$C T$ & $x$ & & & & & & \\
\hline MRI & $\mathrm{X}$ & & & & & & \\
\hline $\begin{array}{l}\text { Constant- } \\
\text { Murley score }\end{array}$ & $\mathrm{X}$ & & & $\mathrm{x}$ & $\mathrm{x}$ & $\mathrm{x}$ & $\mathrm{x}$ \\
\hline woos & $\mathrm{x}$ & & & $x$ & $x$ & $x$ & $x$ \\
\hline oss & $\mathrm{x}$ & & & $\mathrm{X}$ & $\mathrm{x}$ & $x$ & $\mathrm{X}$ \\
\hline$E Q-5 D-5 L$ & $\mathrm{X}$ & & & $\mathrm{X}$ & $\mathrm{x}$ & $\mathrm{X}$ & $\mathrm{X}$ \\
\hline
\end{tabular}

Figure 4

SPIRIT figure. Schedule of enrolment and assessments of the 66 patients who are not included in the radiostereometric analysis study.

\section{Supplementary Files}


This is a list of supplementary files associated with this preprint. Click to download.

- Appendix1.pdf

- Appendix2.pdf

- Appendix3.pdf

- Appendix4.pdf

- Appendx5.pdf

- EQ5D5L.pdf

- Oxfordshoulderscoredanskversion.pdf

- WOOSskema.pdf

- constantshoulderscoreskemadansk.pdf 